\title{
A Long Stroke Electromagnetic $X Y$ Positioning Stage for Micro Applications
}

\author{
Muneeb Ullah Khan, Nabil Bencheikh, Christine Prelle, Frédéric Lamarque, Tobias Beutel, \\ and Stephanus Büttgenbach
}

\begin{abstract}
In this paper, a Lorentz force-based $X Y$ positioning stage with a stack of four electromagnetic linear motors in parallel configuration is presented. The overall design of the positioning stage consists of a mobile and a fixed part separated using a fourpoint contact technique. The uniqueness of the proposed positioning stage lies in its light design with the ability to perform variable strokes at short $(<\mathbf{1 0 0} \mu \mathrm{m})$ and long range (millimeter level) with preembedded auto guidance feature. The analytical modeling and experiment have been realized. The open- and closed-loop performance of positioning stage in linear and nonlinear trajectories have been tested and good agreement is observed between experimental and analytical results. The positioning stage is able to perform variable strokes up till $2 \mathrm{~mm}$ in the $x y$ plane. In closed loop, the maximum precision errors in short and long strokes are found to be 0.031 and $0.451 \mu \mathrm{m}$, respectively. The maximum travel speed is $12 \mathrm{~mm} / \mathrm{s}$ in open loop.
\end{abstract}

Index Terms-Actuators, electromagnetic forces, motion measurement, permanent-magnet motors.

\section{INTRODUCTION}

$\mathbf{I}$ $\mathrm{N}$ recent years, micropositioning systems have been increasingly applied in the field of microelectronic component manufacturing / assembly, biomedicine, scanning microscopy, optical devices, etc., [1]-[4]. In these applications, submicrometer level accuracy, high precision, and repeatability are critical parameters. The said characteristics strongly rely on the design of positioning stages. In general, positioning stages are realized with linear or rotary direct drives [5]. These drives are capable of delivering motion in a workspace with minimum energy loss (e.g., stiction).

In the literature, numerous linear drive technologies (e.g., piezoelectric, electrostatic, etc.,) with dedicated controls have been integrated into positioning stages. These technologies have

Manuscript received January 11, 2011; revised March 22, 2011; accepted April 2, 2011. Recommended by Technical Editor M. Iwasaki. This work is a joint research project between the Université de Technologie de Compiègne, France, and the Technische Universität Braunschweig, Germany.

M. U. Khan is with the Université de Technologie de Compiègne, 60200 Compiègne, France, and also with the Technische Universität Braunschweig, 38106 Braunschweig, Germany (e-mail: muneeb-ullah.khan@utc.fr).

N. Bencheikh is with CEDRAT Technology, F-38243 Meylan, France (email: nabil.bencheikh@cedrat.com).

C. Prelle and F. Lamarque are with the Roberval Laboratory, Université de Technologie de Compiègne, 60200 Compiègne, France (e-mail: christine.prelle@utc.fr; frederic.lamarque@utc.fr).

T. Beutel and S. Büttgenbach are with the Institute for Microtechnology, Technische Universität Braunschweig, 38106 Braunschweig, Germany (e-mail: t.beutel@tu-bs.de; s.buettgenbach@tu-bs.de).

Digital Object Identifier 10.1109/TMECH.2011.2142319 been well studied and implemented with optimal mechanical designs to efficiently redeem different forms of energy for positioning purpose [6]-[11]. However, in particular electromagnetic actuation-based positioning stages are considered to be an acceptable choice when high dynamics with long strokes (millimeter level) are needed in micro applications [12]-[17]. In addition, the recent developments in microfabrication technology have introduced significant reduction in mass and volume of permanent magnets (PMs). These developments have encouraged researcher to build PMs-based miniature designs [18], [19].

In general, a positioning stage consists of drives, mechanical structure (or platform), and feedback sensors. Many physical and technological solutions are possible for each part. With respect to the electromagnetic-based direct drives, fixed current carrying coils with moving-magnet based solution is mostly adapted. This reduces the external disturbances during the functioning of positioning stage, e.g., wires, Joule heating in coils, etc. The moving-magnet part of these drives is often constructed by arranging the PMs in the Halbach configuration to achieve translation and levitation [20]. The motion forces (or Lorentz forces) are generated by placing current carrying coils beneath the moving-magnet part.

In electromagnetic-based positioning stages, the geometrical dimensions of PMs are important parameters. These parameters define the output characteristics of these positioning stages such as travel range and power [21]. Miniaturization of these electromagnetic stages imposes low control signal due to the geometrical limit of the current carrying coils. In order to gain more power, increase in current may damage the coils or degrade the remanent magnetization of the PMs due to the Joule heating effect. Based on our previous work [22], an adjacently inverted north-south (NS) configuration of PMs will be used in this study to overcome these problems. This configuration results in several advantageous phenomena such as symmetrical flux density on both sides of the PM array, a preembedded guidance feature, and a light design as compared to the Halbach configuration [20]. Also, the NS configuration of PMs provides magnetically stable structure that facilitates the easy assembly.

Above all, this paper presents a compact Lorentz force-based $X Y$ positioning stage capable of delivering motion with submicrometer level resolution and millimeter level travel range. The magnetic and mechanical models are described in Section III. The experimental design has been realized by stacking four linear electromagnetic motors to a Zerodur cross structure. To measure the displacement, wireless positioning detection via fiber optic sensors is adopted (see Section IV). Finally, the linear and planar performances in open and closed loops are presented and discussed in Section V. 


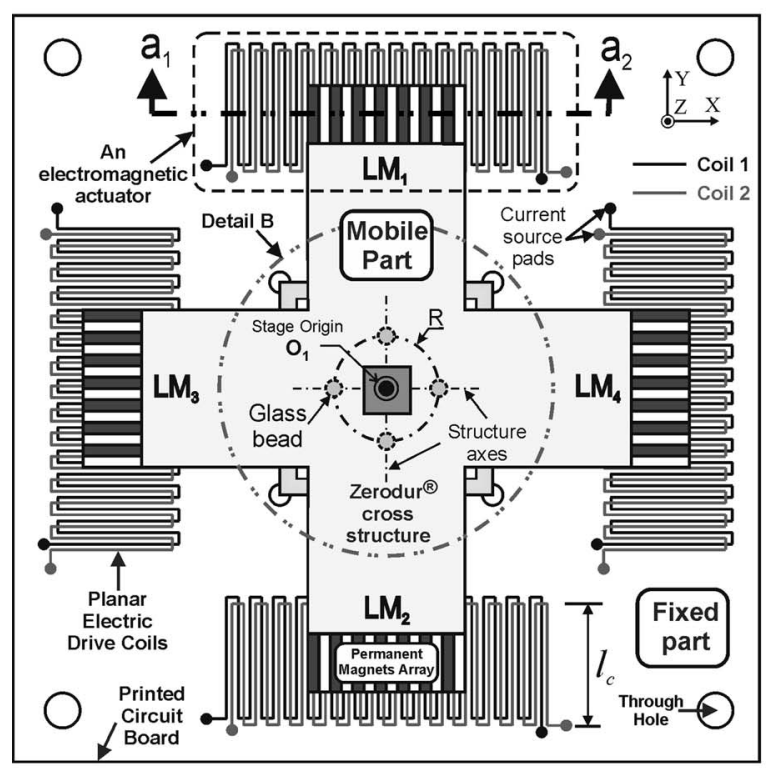

(a)

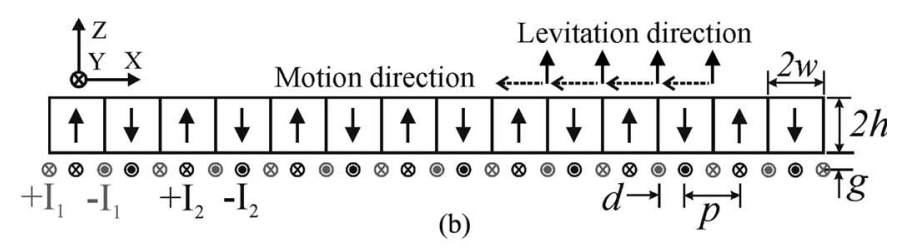

(b)

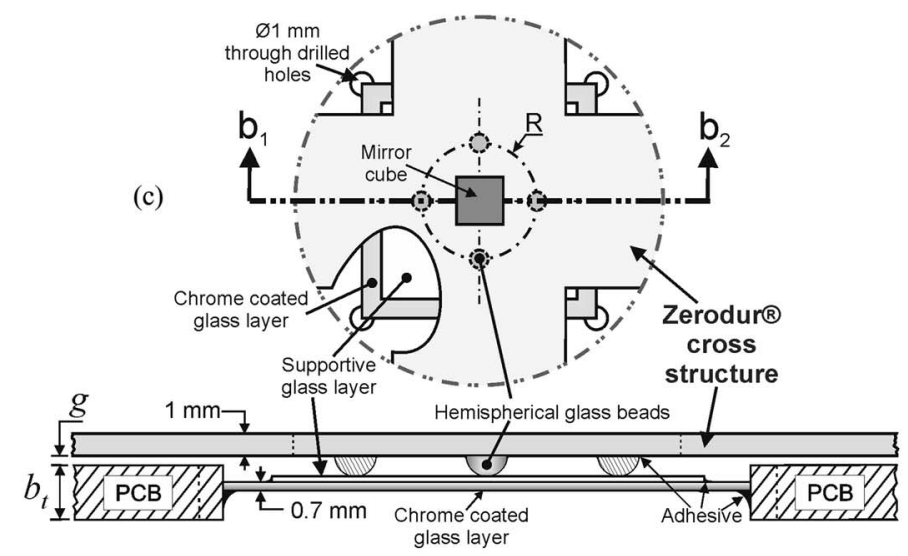

(d)

Fig. 1. (a) Top view of the positioning stage. (b) $a_{1}-a_{2}$ cross-sectional view of $\mathrm{LM}_{1}$. (c) Top view of Detail B. (d) $b_{1}-b_{2}$ cross-sectional view.

\section{PRINCIPLE}

The positioning stage consists of four linear motors (LMs) as shown in Fig. 1(a). Each LM is an electromagnetic actuator based on the Lorentz force principle. Fig. 1(b) represents the cross section of a LM that consists of fixed planar electric drive coils (PEDC) placed beneath the movable permanent-magnets array (PMA). Two sinusoidal currents $I_{1}$ and $I_{2}$ with a relative phase shift of $\pi / 2$ are injected simultaneously into coil 1 and coil 2 of the PEDC [see Fig. 1(a)]. Due to the interaction between magnetic flux density from the PMA and currents in the PEDC, the PMA is subjected to electromagnetic force and, thus, moves along the $x$-axis [see Fig. 1(b)]. The sign of the phase shift between injected currents $I_{1}$ and $I_{2}$ determines the direction of the movement along the $x$-axis. The detailed construction of an LM is provided in [20].

\section{Design Layout AND Description}

The presented positioning stage consists of a fixed and a mobile part as shown in Fig. 1(a). The fixed part includes four PEDCs-fabricated orthogonally onto a double sided $80 \mathrm{~mm} \times$ $80 \mathrm{~mm}$ printed circuit board of $1.5 \mathrm{~mm}$ thickness $b_{t}$. Each PEDC includes a pair of coils (coil 1 and coil 2) with current $I_{1}$ and $I_{2}$ where a positive and negative sign represents inward and outward directions, respectively [see Fig. 1(b)]. The period $p$ of a coil is $1 \mathrm{~mm}$ with copper thickness and width of 35 and $250 \mu \mathrm{m}$, respectively. The length $l_{c}$ of the copper wires is $20 \mathrm{~mm}$ [see Fig. 1(a)]. The air gap $d$ between coil 1 and coil 2 is $500 \mu \mathrm{m}$ as shown in Fig. 1(b). In order to connect the PEDCs to an external power source, current source pads (vias) are fabricated. In addition, a $30 \mathrm{~mm} \times 30 \mathrm{~mm}$ through milled cavity is fabricated in order to affix a $0.7-\mathrm{mm}$ thick one-sided chrome-coated glass layer of the same dimensions as of milled cavity [see Fig. 1(c)]. This glass layer serves as a reflective mirror, which is used to level the fixed part using a laser in the horizontal $x y$ plane about the stage origin $O_{1}$. To reduce the friction between the mobile part and the fixed part, a highly polished $22 \mathrm{~mm} \times 22 \mathrm{~mm} \times$ $0.175 \mathrm{~mm}$ supportive glass layer is installed between the mobile and fixed part [see Fig. 1(d)]. The indirect installation of the supportive glass layer is to avoid deformation due to weight $(82.57 \mathrm{mN})$ of the mobile part.

The mobile part shown in Fig. 1(a) is an assembly of four PMA, a Zerodur cross structure, four hemispherical glass beads and a gold-coated mirror cube.

\section{A. PMA}

One PMA consists of 14 commercially available gold-coated Neodium-Iron-Boron (NdFeB) PM. Each PM is of square cuboid shape with the cross section $(2 w \times 2 h)$ and the length $l_{m}$ of $1 \mathrm{~mm}^{2}$ and $6 \mathrm{~mm}$, respectively. The remanent magnetization of each PM is $1.44 \mathrm{~T}$. The magnetization of PMs in a PMA is periodically oriented along the $z$-axis as shown in Fig. 1(b). This mode of arrangement provides self-assembly, lighter weight, and a symmetrical magnetic flux field on the top and bottom sides of a PMA as concluded in our previous studies [20], [22], [23].

\section{B. Cross Structure}

Four PMAs are assembled orthogonally $50 \mathrm{~mm}$ apart, onto a $50 \mathrm{~mm} \times 50 \mathrm{~mm}$ cross structure of $1 \mathrm{~mm}$ thickness via cyanoacrylate glue. The cross structure is conventionally fabricated in the Zerodur glass material. This material is selected because of its low density $\left(2.53 \mathrm{~g} / \mathrm{cm}^{3}\right)$, high strength $(9.1 \times$ $\left.10^{10} \mathrm{~Pa}\right)$, and good resistance to thermal deformation $(0.05 \pm$ $\left.0.10 \times 10^{-6} / \mathrm{K}\right)$. The geometrical design parameters of the Zerodur cross structure are selected to minimize the overall weight 
and magnetic effect at the center of the cross structure induced by the four PMAs attached to it [20].

In addition, four $0.5-\mathrm{mm}$ diameter hemispherical glass beads are glued to the bottom side of the Zerodur cross structure [see Fig. 1(d)] in a circular array with a radius $R$ of $11 \mathrm{~mm}$. The purpose of these glass beads is to lift the mobile part on four points to create an air gap $g$ and reduce the adherence effect between the mobile and fixed part of the positioning device during motion. A gold-coated mirror cube is installed at the center of cross structure that facilitates in displacement measurement via a noncontact method using fiber optic displacement sensors (see Section IV).

\section{Modeling AND ANALYSiS}

The design of the positioning stage shown in Fig. 1(a) is symmetrical and a pair of LMs along each axis $(x$ - and $y$-axis) contributes to the generation of the driving motion. The currents in the PEDCs are parallel to the length of the PMs in the PMAs along an axis [see Fig. 1(a)]. In order to build an analytical model few assumptions were done for the purpose of simplifications that include perfect orthogonal arrangement of all four PMAs in the $x y$ horizontal plane with no misalignment between the pair of LMs along an axis. In addition, it is assumed that all four PMAs have homogenous magnetic field and there is no deflection in the mobile part of the positioning stage. With these assumptions, the analytical solution for the whole positioning stage is confined by selecting one $\mathrm{LM}$ (e.g., $\mathrm{LM}_{1}$ ), i.e., an assembly of a PMA and a PEDC separated with an air gap $g$ as shown in Fig. 1(b). Afterward the solution is then extended to consider the mechanical behavior of whole stage.

\section{A. Magnetic Flux Density Modeling}

The analytical model of magnetic flux distribution is computed by reducing one PM in a PMA to an equivalent current distribution. It was assumed that there is no variation in magnetization of each PM along the $y$-axis [see Fig. 1(b)]. The magnetic field expression for one PM of width $2 w$ and height $2 h$ in the $x z$ plane is provided in (1) and (2) [24], where $B_{x}^{(1)}$ and $B_{z}^{(1)}$ represent the horizontal and vertical components, respectively, of the external magnetic flux density due to one PM, $M_{s}$ is the magnetization, and $\mu_{0}$ is the magnetic permeability of air

$$
\begin{aligned}
B_{x}^{(1)}(x, z)= & \frac{\mu_{0} M_{s}}{4 \pi}\left[\ln \left(\frac{(x+w)^{2}+(z-h)^{2}}{(x+w)^{2}+(z+h)^{2}}\right)\right. \\
& \left.-\ln \left(\frac{(x-w)^{2}+(z-h)^{2}}{(x-w)^{2}+(z+h)^{2}}\right)\right] \\
B_{z}^{(1)}(x, z)= & \frac{\mu_{0} M_{s}}{2 \pi}\left[a \tan \left(\frac{2 h(x+w)}{(x+w)^{2}+z^{2}-h^{2}}\right)\right. \\
& \left.-a \tan \left(\frac{2 h(x-w)^{2}}{(x-w)^{2}+z^{2}-h^{2}}\right)\right] .
\end{aligned}
$$

Moreover, (3) and (4) represent the total external magnetic flux density components $B_{x}$ and $B_{z}$ due to 14 PMs in a PMA. A very good correlation between analytical the magnetic flux density model and finite element analysis (FEA) results was
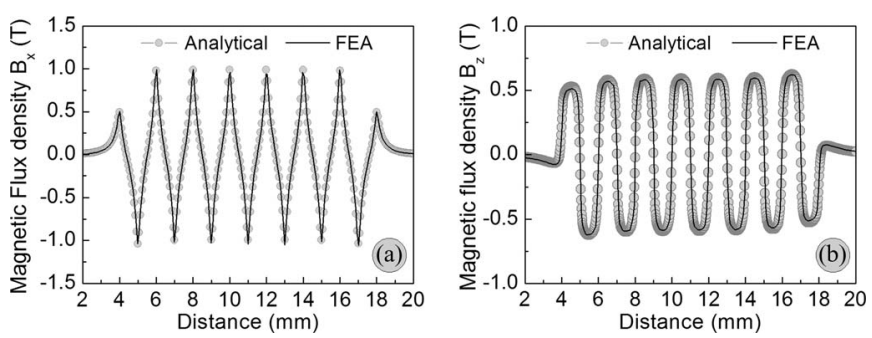

Fig. 2. Analytical and FEA model comparison for (a) $B_{x}$ (b) $B_{z}$.

obtained for one PMA at 60- $\mu$ m air gap (see Fig. 2)

$$
\begin{aligned}
B_{x}(x, z)= & \frac{\mu_{0} M_{s}}{4 \pi}\left[2 \sum_{i=1}^{14} \ln \left(\frac{(x-2 i w)^{2}+(z-h)^{2}}{(x-2 i w)^{2}+(z+h)^{2}}(-1)^{i}\right)\right. \\
& -\ln \left(\frac{(x-28 w)^{2}+(z-h)^{2}}{(x-28 w)^{2}+(z+h)^{2}}\right) \\
- & \left.\ln \left(\frac{(x+w)^{2}+(z-h)^{2}}{(x+w)^{2}+(z+h)^{2}}\right)\right] \\
B_{z}(x, z)= & \frac{\mu_{0} M_{s}}{4 \pi}\left[2 \sum_{i=1}^{14} a \tan \left(\frac{2 h(x-2 i w)(-1)^{i}}{(x-2 i w)^{2}+z^{2}-h^{2}}\right)\right. \\
& -a \tan \left(\frac{2 h(x-28 w)}{(x-28 w)^{2}+z^{2}-h^{2}}\right) \\
& \left.-a \tan \left(\frac{2 h x}{(x-2 w)^{2}+z^{2}-h^{2}}\right)\right]
\end{aligned}
$$

\section{B. Electromagnetic Force Computation}

The electromagnetic force (or Lorentz force) generated by one LM was computed using a general (5) [24], where $B_{\text {ext }}$ is the external magnetic flux density component $B_{x}$ and $B_{z}$ computed using (3) and (4). The superscript $k$ denotes the relative position index for magnetic flux density computation with respect to coil 1 and coil 2 in a PEDC. $N$ denotes the number of turns in a coil.

$$
F_{\text {electromagnetic }}=N \sum_{k=1}^{2} I_{k} \int_{\text {wire }} d l \times B_{\text {ext }}^{(k)} \text {. }
$$

In order to identify the magnitude of electromagnetic force component $F_{y}$ along the $y$-axis [see Fig. 1(b)], a static analysis was performed at 100- $\mu \mathrm{m}$ air gap $g$ in semianalytical software RADIA. The analysis revealed that for a maximum allowable current of $0.8 \mathrm{~A}$ per coil (limited due to the dimensions of PEDCs) the magnitude of electromagnetic force $F_{y}$ generated by $\mathrm{LM}_{1}$ in the $x y$ plane is very small $\left(6.89 \times 10^{-10} \mathrm{mN}\right)$. Indeed, the currents in PEDC are parallel to the length of PMA, so the effect of this force is negligible as compared to $F_{x}\left(F_{z}\right)$, which was computed to be $35.89 \mathrm{mN}(55.16 \mathrm{mN})$, respectively. The total electromagnetic force $F_{\text {total }}$ generated along an axis is the sum of the electromagnetic forces generated by LMs along the same axis. 

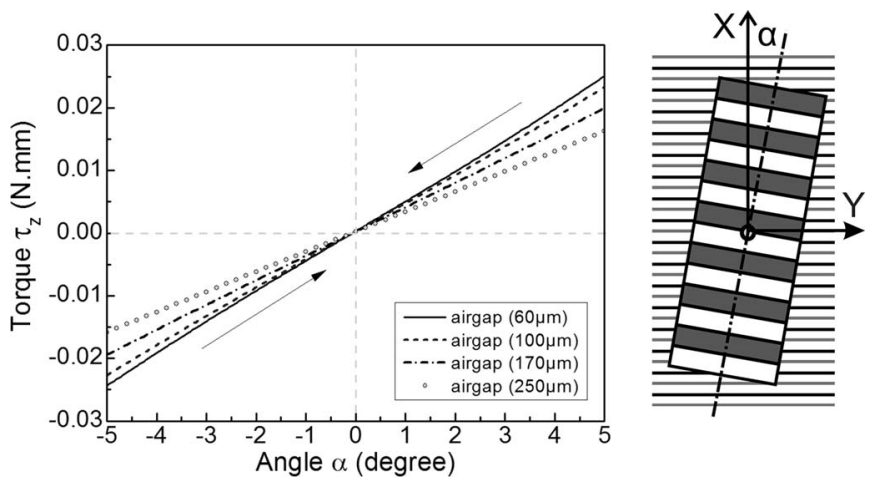

Fig. 3. Torque $\tau_{z}$ due to the misalignment $\alpha$ between PMA and PEDC.

\section{Auto Guidance Effect}

During motion the PMA of an LM (e.g., $\mathrm{LM}_{1}$ ) tends to align itself with respect to PEDC. If a relative misalignment between PMA and PEDC appears, a torque $\tau_{z}$ about the center of the PMA [20] is generated due to the uneven magnetic flux distribution over a length of a current carrying copper wire of the PEDC. In order to compute this torque an analysis was performed in RADIA by rotating the PMA at small angle $\left(\alpha= \pm 5^{\circ}\right)$ in the $x y$ plane with respect to PEDC in $\mathrm{LM}_{1}$. The computed torque $\tau_{z}$ about the $z$-axis of $\mathbf{L M}_{1}$ is shown in Fig. 3. From analysis, it is evident that the magnitude of the torque $\tau_{z}$ is proportional to the angle $\alpha$ in the $x y$ plane and air gap $g$ between PMA and PEDC.

Moreover, when a constant current $\left(I_{1}=I_{2}\right)$ is injected into both coils of a PEDC an electromagnetic force appears over each period of PMA. The magnitude of these electromagnetic forces are same but opposite in direction to each other, which is due to the adjacent NS orientation of the PMs. These forces lock the PMA (moving-magnet part of LM) at a position. This preembedded feature is used to align the mobile part of the positioning stage with respect to the fixed part by injecting constant current along an axis [20]. During experimentation this feature is used to achieve initial condition of the mobile part. In linear travel, this preembedded feature is used to reduce the straightness error by injecting constant currents into the PEDCs situated parallel to the motion axis [22].

\section{Mechanical Model}

A general analytical solution for the translation of the position stage along the $x$ - or $y$-axis is developed in this section. Due to the symmetry of the positioning stage, the total force $F_{\text {total }}$ along an axis is the sum of the generated electromagnetic force. In addition, the stage is supported on glass beads, which develops a friction force between the mobile and fixed part of the positioning stage. While neglecting the thermal and hysteresis effect as disturbances, Newton's second law is used to develop the general solution as given in (6), where $M$ is the total mass of the mobile part, $F_{O_{1}}$ is the residual translation force about stage origin $O_{1}$ and $F_{r}$ is the resistive force

$$
\sum \vec{F}_{O_{1}}=\vec{F}_{\text {total }}-\vec{F}_{r}=M \frac{d^{2} \vec{X}_{R}}{d t^{2}} .
$$

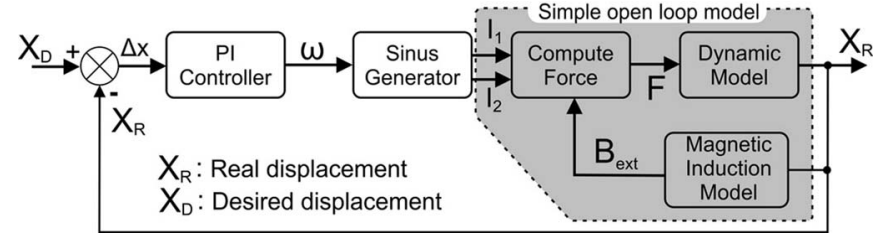

Fig. 4. PI control for one LM.

The resistive force $F_{r}$ is defined as the friction and adhesion force $\left(W \times \mu_{\text {friction }}\right)$ in the translation of the mobile part. The adhesion force was computed using the net weight ( $W_{\text {net }}=$ $W-F_{z}$ ) of the mobile part and adhesion coefficient $\mu_{\text {adhesion }}$, $\left(F_{\text {adhesion }}=W_{\text {net }} \times \mu_{\text {adhesion }}\right)$. If the total force value enters in the range of $\left[-\mathrm{F}_{\text {adhesion }},+\mathrm{F}_{\text {adhesion }}\right]$ its value is set to zero. The friction coefficients $\left(\mu_{\text {friction }}=0.47, \mu_{\text {adhesion }}=0.43\right)$ were experimentally measured using an inclined plane technique. The displacement $X_{R}$ is obtained by double integration of acceleration in (6).

\section{E. Control Model}

At first, an open-loop model has been realized that takes into account both the electromagnetic (5) and mechanical effects (6) during the translation of the positioning stage (see subpart of Fig. 4). The sinusoidal currents $I_{1}=I_{o} \sin \left(\omega t \pm \phi_{1}\right)$ and $I_{2}=$ $I_{o} \sin \left(\omega t \pm \phi_{2}\right)$ with a phase difference $\left(\Delta \phi=\phi_{2}-\phi_{1}\right)$ of $\pi / 2$ are injected into the PEDC of an LM. $I_{o}$ and $\omega$ represent the amplitude and frequency of the injected currents, respectively. In order to achieve reciprocating motion, the sign of the phase difference is changed to $+\pi / 2$ or $-\pi / 2$ [20].

For precise positioning a PI controller was used to control the frequency $\omega$ of the injected sinusoidal currents $I_{1}$ and $I_{2}$ in the PEDCs relative to the displacement difference $(\Delta x=$ $X_{D}-X_{R}$ ) [20]. The PI control algorithm is provided in (7), where $K_{P}$ and $K_{I}$ are the proportional and integral gains of the controller, respectively,

$$
\omega=2 \pi \cdot\left|K_{P} \cdot \Delta x+K_{I} \int_{t_{0} \rightarrow t_{1}} \Delta x d t\right| .
$$

The PI controlled functional block diagram for one LM is shown in Fig. 4. In this manner, a pair of LMs along each axis is controlled simultaneously to achieve rectilinear and nonlinear trajectories.

\section{EXPERIMENTATION}

At first, the real magnetic flux density was measured using a Hall sensor (Allegro-Microsystems, Inc., A1301) by running it parallel to the PMA surface of $\mathrm{LM}_{1}$ along the cross section $a_{1}-a_{2}$ [see Fig. 1(a)]. The Hall sensor used in this experiment only measures the $B_{z}$ component of magnetic flux density due to its working principle. A 200- $\mu \mathrm{m}$ air gap $g$ between the Hall sensor and PMA surface was selected to avoid any physical contact. The sensitivity of the Hall sensor was measured to be $2.44 \mathrm{mV} /$ Gauss. A comparative analysis between the measured magnetic flux density $B_{z}$ and that calculated via the FEA model 


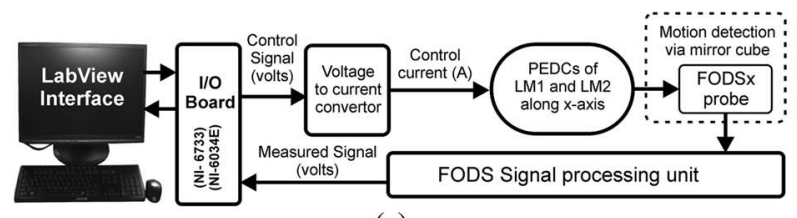

(a)

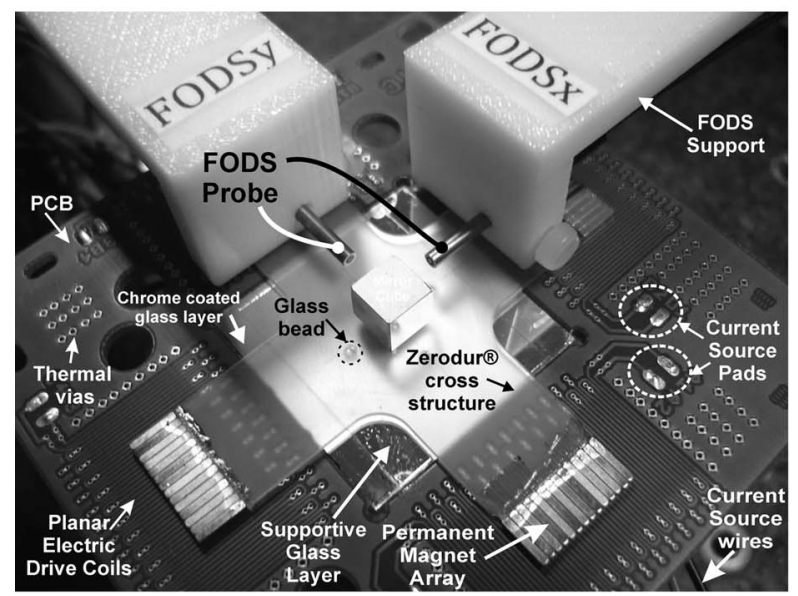

(b)

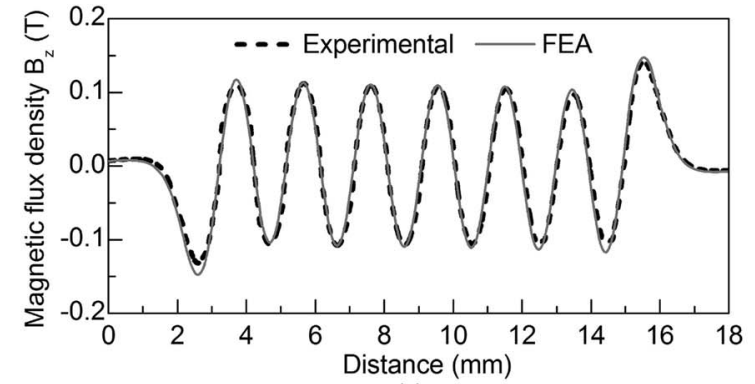

(c)
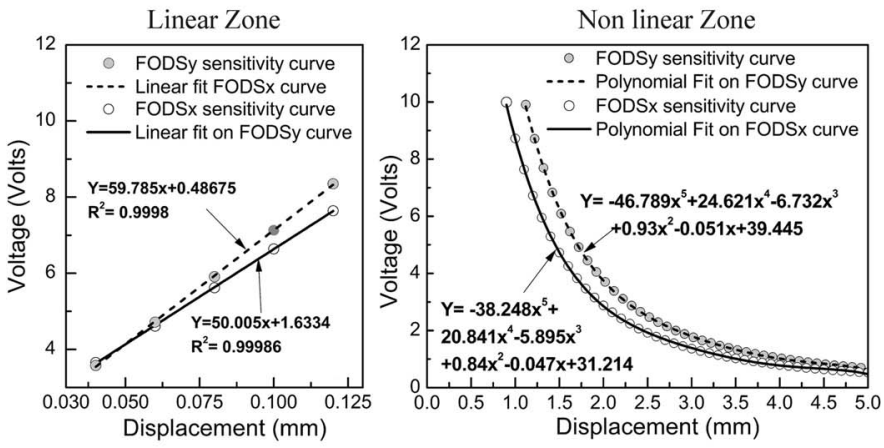

(d)

Fig. 5. (a) Block diagram of hardware configuration along the $x$-axis. (b) Experimental setup. (c) Measured magnetic flux density component $B_{z}$ of LM 1 . (d) FODS sensitivity curves in linear and nonlinear zone.

is shown in Fig. 5(c). The variation in the experimental results is due to the uneven dimensions and magnetization of the PMs assembled in a PMA. However, the results show good agreement and validate the magnetic flux density model (4).

\section{A. Displacement Sensor}

In order to measure the real displacement $X_{R}$ during experimentation, a contactless technique was adapted to avoid external disturbances due to feedback sensor operation. Two fiber optic displacement sensors (FODS) were installed in the experiment (one for each axis) as shown in Fig. 5(b). Each FODS probe consists of a bundle of five fibers (one emission fiber and four reception fibers). The emission fiber (located at center) emits light on a flat mirror target. The reflected light is received by the reception fibers situated around the emission fiber and translated into voltage. The voltage resides in the range of $[1 \mathrm{~V}, 10 \mathrm{~V}]$. The maximum output voltage of FODS is limited due to the data acquisition I/O board specifications. The amount of reflected light is a function of the linear displacement between the sensor probe and the flat mirror placed perpendicular to the FODS probe. The FODS used in this experiment provides a linear zone and a nonlinear zone. The FODS working principle used in this experiment is provided in [25].

\section{B. Experimental Setup}

An experimental setup has been realized as shown in Fig. 5(b). Each LM has two inputs and one output. The functional block diagram of the configuration adopted along the $x$-axis is shown in Fig. 5(a). The analog-digital converter (NI 6034E) and digitalanalog converter (NI 6733) are 16-bit high resolution data ac- quisition I/O boards with two and eight channels, respectively. A sampling time of $10 \mathrm{~ms}$ provided a better behavior of the stage. A pair of voltage to current convertors (sampling rate $50 \mathrm{kHz}$ and power $30 \mathrm{~W}$ each) were used to drive an axis of the positioning stage. A LabView interface was created to control four LMs.

At first, the fixed part was leveled in the horizontal $x y$ plane using a laser- and chrome-coated glass layer that was fixed at the center of the fixed part. The mobile part was then placed on the fixed part and constant currents $\left(I_{1}, I_{2}\right)$ were injected into the PEDCs along the $x$-axis to align the mobile part with respect to PEDCs of the fixed part. In next step, the FODS $x$ and FODS $y$ were sequentially calibrated with the help of a goldcoated mirror cube situated at the center of the cross structure. The data acquisition frequency was $100 \mathrm{~Hz}$. Fig. 5(d) represents the sensitivity curves for linear and nonlinear zones of FODS $x$ and FODSy. The functional range was found to be $80 \mu \mathrm{m}$ in the linear zone and $2.5 \mathrm{~mm}$ in the nonlinear zone for FODS $x$ and FODS $y$. The variation in the sensitivity curves of FODS $x$ and FODSy is mostly due to the electronics of the sensors. In the linear zone, the resolution was computed to be 8 and $11 \mathrm{~nm}$ for FODS $x$ and FODS $y$, respectively. The linear zone was used to evaluate the resolution and precision performances of the stage, and the nonlinear zone was used to validate the millimeter level stroke.

\section{RESULTS AND DISCUSSION}

In this section, the experimental results are discussed. At first, the open-loop tests were conducted to validate the analytical model and then the closed-loop tests were realized. 
This article has been accepted for inclusion in a future issue of this journal. Content is final as presented, with the exception of pagination.

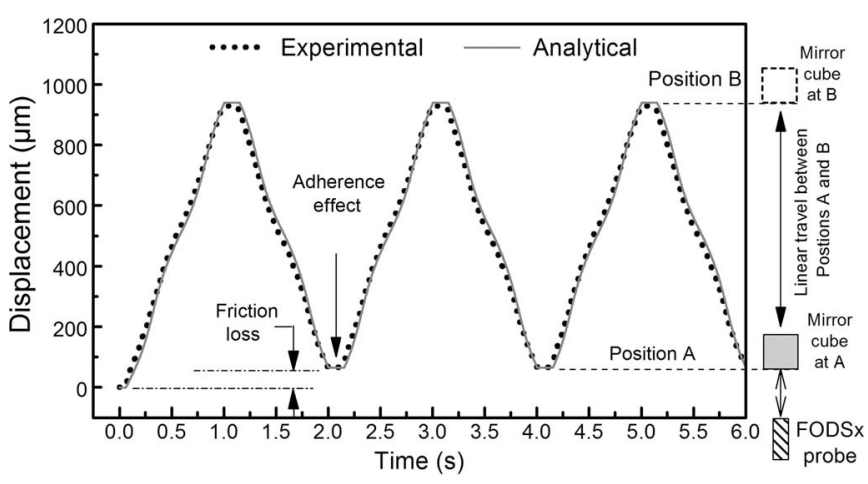

Fig. 6. Numerical and experimental results in open loop.

\section{A. Open-Loop Performance}

1) Displacement: The simulated response of the positioning device in open loop was computed in MATLAB Simulink using the analytical model (6). Experimentally, a period of $1-\mathrm{Hz}$ sinusoidal currents $\left(I_{1}\right.$ and $\left.I_{2}\right)$ with 0.8 A (peak-to-peak) amplitude was injected into the PEDCs situated perpendicular to the motion axis. The sampling frequency of injected currents was $100 \mathrm{~Hz}$ and the FODS was used in the nonlinear zone to measure the long range axial displacement. During linear travel a constant current of $0.8 \mathrm{~A}$ was injected into the PEDCs situated parallel to the motion axis to minimize drift error [22].

A comparative analysis between 3 cycles of simulated and experimental displacement results is shown in Fig. 6. The simulated and experimental positions A (B) were found to be $66.67 \mu \mathrm{m}(955.85 \mu \mathrm{m})$ and $65.01 \mu \mathrm{m}(928.59 \mu \mathrm{m})$, respectively. The large difference between experimental and simulated results at position $\mathrm{B}$ is because of the FODS low sensitivity at position $\mathrm{B}$ [25]. The friction loss for the simulated and experimental results was computed to be 68.21 and $55.41 \mu \mathrm{m}$, respectively. The difference between simulated and experimental results can be explained by the microrotation phenomenon that occurs during the displacement. These microrotations are due to the generation of nonhomogenous electromagnetic forces between a pair of LMs along the axis. Due to this a small offset appears between the generated electromagnetic forces with respect to the structure axes [see Fig. 1(a)] during motion. This results in a microrotation about the center of the stage $O_{1}$. The nonhomogenous electromagnetic force generation is due to the nonidentical magnetic flux density distribution between a pair of PMAs along the axis. This variation is difficult to be avoided in commercial magnets as it highly depends on geometrical tolerances and fabrication process of PMs. In order to reduce this effect, individual control of each LM was used [21]. However, the experimental results provide a good correlation with the simulated results and, thus, validates the analytical model.

2) Position Repeatability: In order to measure the repeatability of the positioning stage a 10-cycles test was performed in similar fashion as discussed in previous section. For a mean displacement of $854.54 \mu \mathrm{m}$, the position repeatability error was calculated to be $4.41 \mu \mathrm{m}$. The relative error was computed to be $3.03 \%$.
TABLE I

Velocity Response of Positioning StAGE

\begin{tabular}{c|c|c|c}
\hline $\begin{array}{c}\text { Control signal } \\
\text { Frequency (Hz) }\end{array}$ & $\begin{array}{c}\text { Theoretical } \\
\text { Velocity }(\mathbf{m m} / \mathbf{s})\end{array}$ & $\begin{array}{c}\text { Measured } \\
\text { Velocity }(\mathbf{m m} / \mathbf{s})\end{array}$ & $\begin{array}{c}\text { Relative } \\
\text { Error }(\%)\end{array}$ \\
\hline 0.5 & 1 & $1.013 \pm 0.002$ & 1.3 \\
1 & 2 & $2.016 \pm 0.017$ & 0.8 \\
2 & 4 & $4.007 \pm 0.011$ & 0.175 \\
4 & 8 & $8.006 \pm 0.041$ & 0.075 \\
5 & 10 & $9.954 \pm 0.086$ & 0.46 \\
6 & 12 & $11.088 \pm 0.149$ & 7.6 \\
\hline
\end{tabular}

3) Straightness: In order to study the straightness error in long strokes both FODS feedback sensors were used simultaneously. The axial displacement (along the $x$-axis) and lateral displacements (along the $y$-axis) were measured using the FODS $x$ and FODSy, respectively [see Fig. 5(b)]. The FODS $x$ was used in the nonlinear zone and FODSy was used in its linear zone. The injected current characteristics were the same as described in the displacement section. However, to investigate the effect of travel range two tests were performed for one period and half period of 1-Hz sinusoidal currents [see Fig. 7(a) and (c)]. The lateral displacement is magnified by 100 for the ease of observation. The straightness errors are not only because of the lateral displacement, but also due to the manual assembly of mirror cube onto the mobile part, the mirror cube surface is not parallel to the FODSy. The angle between the displacement axis and the mirror cube surface facing FODS $y$ was calculated to be $0.92^{\circ}$. As the FODSy is in its linear zone so the real straightness was computed by subtracting the error due to the mirror angle. The straightness for $500 \mu \mathrm{m}(1000 \mu \mathrm{m})$ displacement was calculated to be $1.991 \mu \mathrm{m}(2.353 \mu \mathrm{m})$ with a standard deviation of $0.460 \mu \mathrm{m}(0.568 \mu \mathrm{m})$, respectively, [see Fig. 7(b) and (d)].

4) Velocity Response: The velocity response was measured along the $x$-axis using FODS $x$. Different control signal frequencies of injected currents $I_{1}$ and $I_{2}$ were used [see Fig. 7(e)]. The experimentally measured velocities with theoretical values for 10 cycles of linear travel are provided in Table I. The variation in the experimentally measured velocities are due to several phenomena that occur during translation (e.g., microrotations, etc.). From the results it is evident that the static error in the velocity is due to the inertia of the mobile part as it is directly proportional to the velocity. The maximum velocity achieved was $12 \mathrm{~mm} / \mathrm{s}$ in the 10-cycle linear travel test. This limitation is due to the inertia of the mobile part.

5) XY Linear Profile: In order to study the $x y$ displacement of the positioning stage, all four LMs were used simultaneously. Both FODS $x$ and FODS $y$ were used in their nonlinear zone to measure the long stroke $x y$ linear displacement. A 5-cycle test of linear trajectory in the $x y$ plane was performed by injecting one period of $1-\mathrm{Hz}$ sinusoidal currents with $0.8 \mathrm{~A}$ per coil (peakto-peak) amplitude. The trajectory is repeatable from position A to B and vice versa [see Fig. 7(f)]. However, the hysteresis in the curve is due to the torque effect that is generated due to the assembly error [22], which forces the mobile part to acquire different trajectory in backward travel (from position B to A). The hysteresis was calculated to be $57 \mu \mathrm{m}$. 

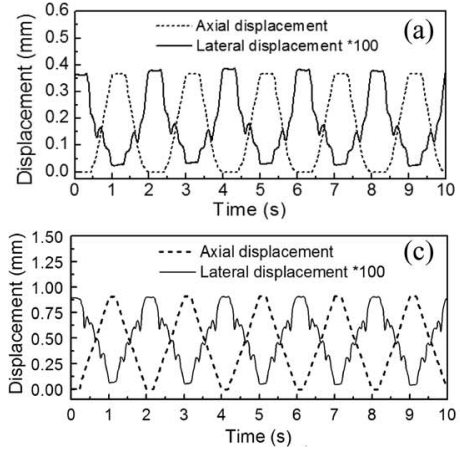
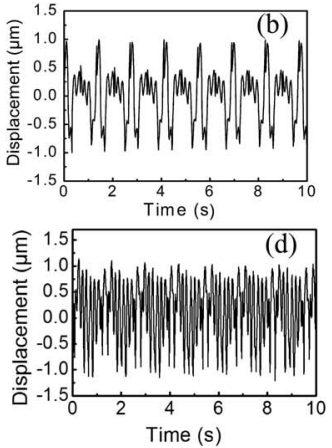
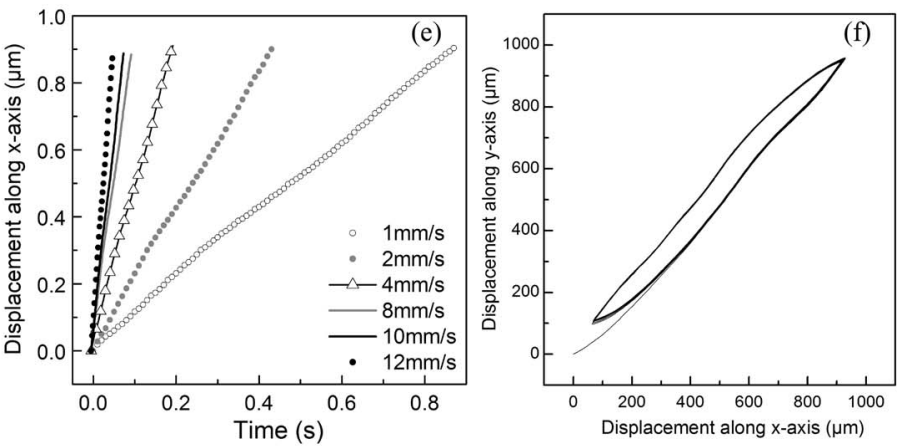

Fig. 7. (a) $x$ - and $y$ - axis response for the half period of injected sinusoidal current. (b) Straightness error for the half period of injected sinusoidal current. (c) $x$ and $y$ - axis response for the one period of injected sinusoidal current. (d) Straightness error for the one period of injected sinusoidal current. (e) Velocity response along the $x$-axis. (f) $x y$ linear displacement in open loop.
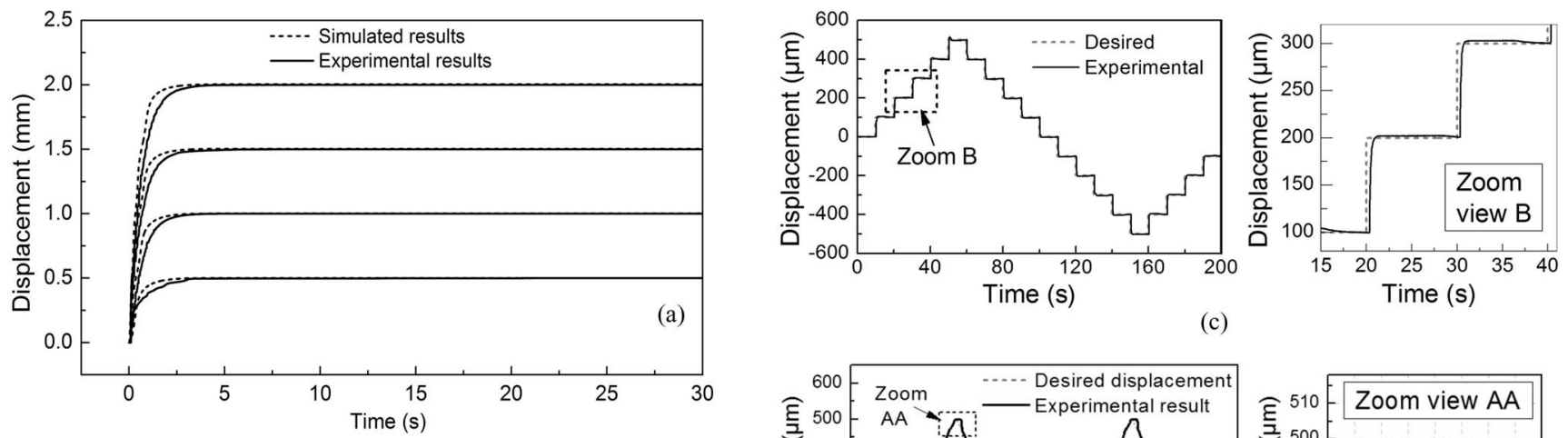

(c)
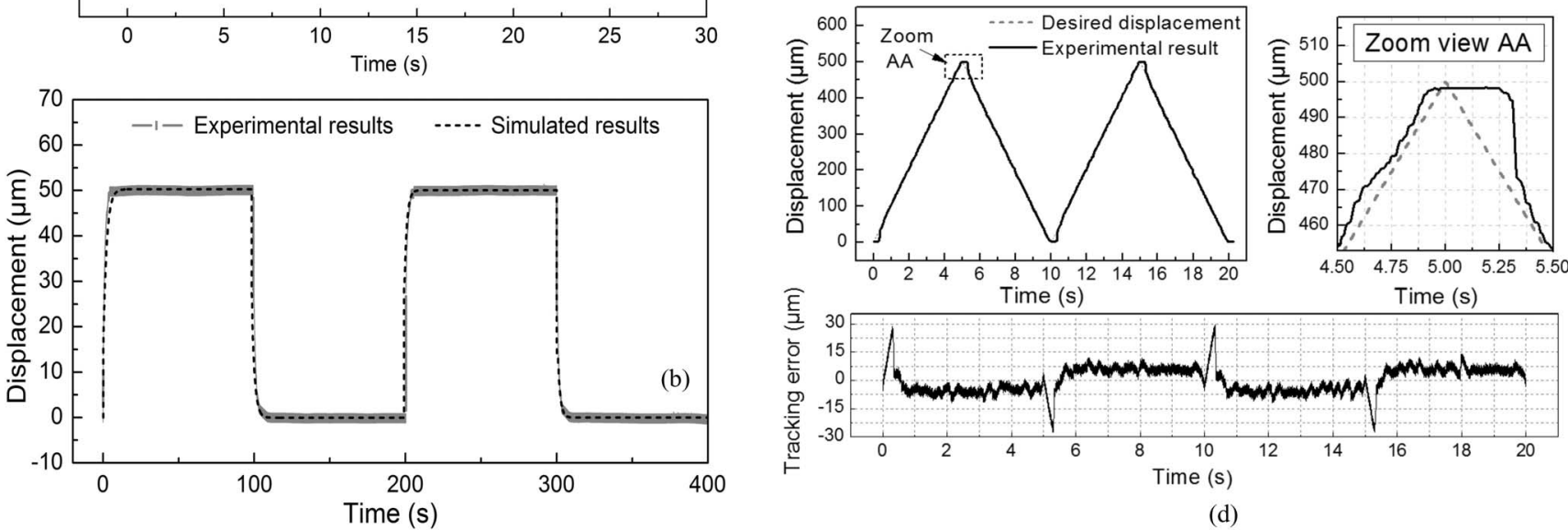

(d)

Fig. 8. (a) Long stroke step response. (b) Short stroke step response. (c) Step train response. (d) Triangular response with tracking error plot.

\section{B. Closed-Loop Performance}

In this section, the positioning stage has been controlled via a PI controller. The desired displacement was achieved by changing the frequency $\omega$ of the controlling currents with respect to the error computed between desired and real displacement. The selection of PI controller gains depends on several parameters such as design, dynamics of the positioning stage as well as on the sensitivity of the FODS. To identify the controller gains ( $K_{P}$ and $K_{I}$ ), the linear zone of the FODS has been used as the sensitivity remains constant in this zone.

1) Square Step Response: Initially, both axes were configured separately for a 50- $\mu \mathrm{m}$ step response and then the same gain values were used for long stroke step response (millimeter level). Very good agreement between simulated and experimental results along the $x$-axis was obtained [see Fig. 8(a) and (b)].
TABLE II

REPEATABILITY IN 10-CyCLE SQUARE STEPS TEST

\begin{tabular}{c|c|c}
\hline $\begin{array}{c}\text { Desired } \\
\text { displacement }(\mu \mathbf{m})\end{array}$ & $\begin{array}{c}\text { Simulated } \\
\text { displacement }(\mu \mathbf{m})\end{array}$ & $\begin{array}{c}\text { Measured } \\
\text { displacement }(\mu \mathbf{m})\end{array}$ \\
\hline 50 & 50.265 & $49.975 \pm 0.031$ \\
500 & 500.930 & $499.793 \pm 0.268$ \\
1000 & 1000.302 & $999.815 \pm 0.309$ \\
1500 & 1499.325 & $1500.387 \pm 0.451$ \\
2000 & 1999.855 & $2000.174 \pm 0.360$ \\
\hline
\end{tabular}

$K_{P}$ and $K_{I}$ gain values have been selected to avoid any overshoot, which is a critical criterion in most of the micro applications, for example, surface scanning or microassembly. From the results it is evident that the closed-loop model (see Fig. 4) developed in Section III can be used to compare the experimental results. Indeed, there are several uncertainties that change 


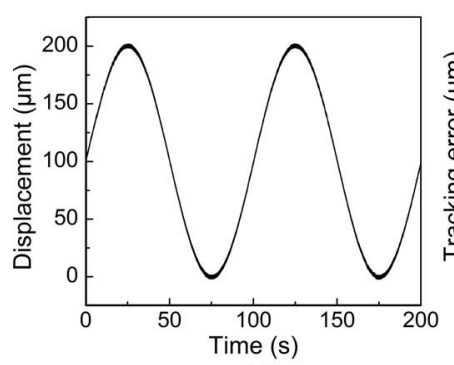

(a)

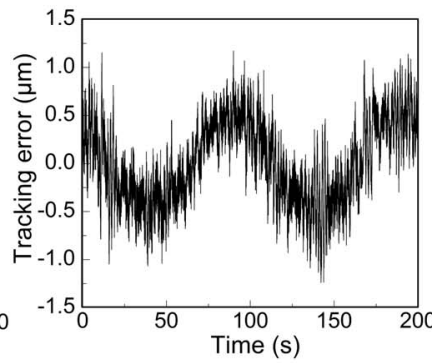

(b)

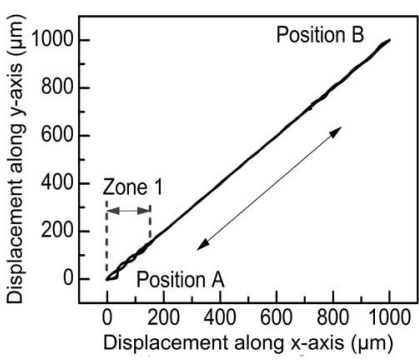

(c)

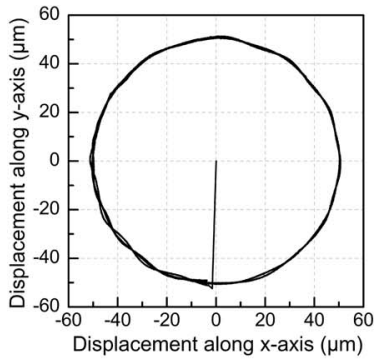

(d)

Fig. 9. (a) Sinusoidal displacement. (b) Tracking error in sinusoidal displacement. (c) $x y$ linear displacement. (d) Circular profile in closed loop.

the output of the positioning stage. For example, microrotations during linear travel can change the sensitivity of the FODS, impurities (such as dust) can change the sticking and friction force, etc. These uncertainties require complex multiphysics analyses and significant computational time, programming, and experimentation; thus, global gain values for whole positioning stage cannot be used in this scenario. However, an approximation was adapted by selecting different gain values $\left(K_{P}=0.077\right.$, $K_{I}=0.010$ for the $x$-axis and $K_{P}=0.085, K_{I}=0.012$ for the $y$-axis) to realize the experiment. The average settling time was computed to be $0.921 \mathrm{~s}(5 \%)$ for long strokes and $0.256 \mathrm{~s}$ (5\%) for short stroke [see Fig. 8(a) and (b)]. Moreover, a 10 -cycle square step test was conducted along the $x$-axis, to evaluate the repeatability of the positioning stage in closed loop. The experimental and simulated results are provided in Table II. The variation in the simulated and the experimental displacements are mainly due to the adhesion effect and fixed controller gain values.

2) Step Train Response: A step train motion is one of the most common operations of the positioning stages used in micro applications. In the semiconductor industry, for example, it is used in order to achieve high-quality processing in products such as ICs. Therefore, in order to validate this characteristic an experiment along the $x$-axis, with each step equal to $100 \mu \mathrm{m}$ and moving toward $\pm 0.5 \mathrm{~mm}$ has been conducted. Very good agreement was observed among the experimental and desired results as shown in Fig. 8(c). The average settling time (5\%) for $100 \mu \mathrm{m}$ in continuous step was measured to be $306.2 \mathrm{~ms}$.

3) Triangular Response: To illustrate the tracking ability of the positioning stage, a triangular profile test has been performed. The positioning stage was guided along the $x$-axis with the desired triangular profile of $0.5-\mathrm{mm}$ amplitude. The experimental results show very good agreement as shown in Fig. 8(d). However, the friction effect can be seen as shown in the zoom view of the Fig. 8(d). From tracking error plot, the magnitude of this friction effect was computed to be $28.38 \mu \mathrm{m}$. In the time interval [1 s, $5 \mathrm{~s}$ ], the positioning stage starts moving and the sticking force value falls to $5.63 \mu \mathrm{m}$ due to the dynamics of the positioning stage. Moreover, Fig. 8(d) reveals that the rms tracking error in zone $[1 \mathrm{~s}, 5 \mathrm{~s}]$ remains in the range of $5.134 \mu \mathrm{m}$ with a standard deviation of $\pm 0.929 \mu \mathrm{m}$. These performances can be improved with the help of more appropriate controller.

4) Sinusoidal Response: A sinusoidal displacement of 200- $\mu \mathrm{m}$ (peak-to-peak) amplitude was achieved along the $x$-axis [see Fig. 9(a)]. The sampling frequency of the injected currents was $0.01 \mathrm{~Hz}$. The tracking error was computed to be $1.206 \mu \mathrm{m}$ with a standard deviation of $0.416 \mu \mathrm{m}$ [see Fig. 9(b)].

5) XY Linear profile: To achieve planar performances of the positioning stage all four LMs are operated simultaneously. Fig. 9(c) represents a 5-cycle test for 1-mm linear $x y$ travel. The average linear displacement was found to be $1.039 \mathrm{~mm}$ with a standard deviation of $\pm 0.937 \mu \mathrm{m}$. The chaotic behavior in zone 1 in Fig. 9(c) is due to the friction effect. As the displacement error $\Delta x$ falls to zero, the magnitude of the current also reduces in the PEDCs and, thus, the relative value of adhesion forces increases with respect to the generated electromagnetic force. This results in a variable force along an axis. This difference in force value generates microtorques about stage origin $O_{1}$. An adaptive PID controller can be used to change the controller gain values with respect to $\Delta x$. However, the open-loop hysteresis effect is eliminated.

6) Circular Profile Performance: A circular profile of $100 \mu \mathrm{m}$ in diameter was achieved in similar fashion as explained in the previous paragraph. Fig. 9(d) represents three circular trajectories at $0.1 \mathrm{~Hz}$. The average tracking error value was calculated to be $2.459 \mu \mathrm{m}$ with a standard deviation of $\pm 0.831 \mu \mathrm{m}$.

In the end, the load carrying capacity of the positioning stage was tested by placing small weights at the center of the mobile part. The maximum load carrying capacity was found to be $11.073 \mathrm{~g}$ for the maximum allowable current of $0.8 \mathrm{~A}$ (peakto-peak) amplitude to avoid Joule heating in the PEDCs. There are many possibilities to increase this capacity for example reducing the air gap $g$ between the PMAs and PEDCs, using stronger miniature magnets, and reducing the weight of the cross structure.

\section{CONCLUSION AND FUtURE WORK}

A multifeatured electromagnetic-based $X Y$ positioning stage design has been presented. The short and long stroke performances in open- and closed-loop control have been evaluated and compared with analytical results. The positioning stage is able to perform variable strokes up to $2 \mathrm{~mm}$ (limited due to FODS size)In closed loop, the maximum precision errors in short and long strokes are found to be 0.031 and $0.451 \mu \mathrm{m}$, respectively. The maximum travel speed was found to be $12 \mathrm{~mm} / \mathrm{s}$ in open loop. 
The rotation of the positioning stage about the $z$-axis could be achieved by individual control of each LM in closed loop. In future, the PI control algorithm will be modified to analyze the rotation behavior of the positioning stage. In addition, the mobile part of the positioning stage will be fabricated using microfabrication technology to facilitate the easy assembly of PMs.

\section{REFERENCES}

[1] S. Polit and J. Dong, "Development of a high-bandwidth xy nanopositioning stage for high-rate micro-/nanomanufacturing," IEEE/ASME Trans. Mechatronics, vol. 16, no. 4, pp. 724-733, Aug. 2011.

[2] D. Robert, D. Fayol, C. Le Visage, G. Frasca, S. Brulé, C. Méenager, F. Gazeau, D. Letourneur, and C. Wilhelm, "Magnetic micromanipulations to probe the local physical properties of porous scaffolds and to confine stem cells," Biomaterials, vol. 31, no. 7, pp. 1586-1595, 2010.

[3] S. H. Jeong, G. H. Kim, and K. R. Cha, "A study on optical device alignment system using ultra precision multi-axis stage," J. Mater. Process. Technol., vol. 187-188, pp. 65-68, 2007.

[4] D. Kang, K. Kim, D. Kim, J. Shim, D.-G. Gweon, and J. Jeong, "Optimal design of high precision xy-scanner with nanometer-level resolution and millimeter-level working range," Mechatronics, vol. 19, no. 4, pp. 562 570, 2009.

[5] P. Ouyang, R. Tjiptoprodjo, W. Zhang, and G. Yang, "Micro-motion devices technology: The state of arts review," Int. J. Adv. Manuf. Technol., vol. 38, no. 5, pp. 463-478, 2008.

[6] L. Yangmin and X. Qingsong, "Development and assessment of a novel decoupled $X Y$ parallel micropositioning platform," IEEE/ASME Trans. Mechatronics, vol. 15, no. 1, pp. 125-135, Feb. 2010.

[7] M. Rakotondrabe, Y. Haddab, and P. Lutz, "Development, modeling, and control of a micro-/nanopositioning 2-dof stick-slip device," IEEE/ASME Trans. Mechatronics, vol. 14, no. 6, pp. 733-745, Dec. 2009.

[8] D. Jingyan and P. M. Ferreira, "Electrostatically actuated cantilever with SOI-MEMS parallel kinematic stage," J. Microelectromech. Syst., vol. 18, no. 3, pp. 641-651, 2009.

[9] R. Merry, N. de Kleijn, M. van de Molengraft, and M. Steinbuch, "Using a walking piezo actuator to drive and control a high-precision stage," IEEE/ASME Trans. Mechatronics, vol. 14, no. 1, pp. 21-31, Feb. 2009.

[10] Y. Tian, B. Shirinzadeh, and D. Zhang, "Design and dynamics of a 3DOF flexure-based parallel mechanism for micro/nano manipulation," Microelectron. Eng., vol. 87, no. 2, pp. 230-241, 2010.

[11] J.-C. Lee and D.-W. Lee, "Fabrication of a micro xy-stage using SU-8 thermal actuators," Microelectron. Eng., vol. 86, no. 4-6, pp. 1267-1270, 2010.

[12] C. Mei-Yung, H. Hsuan-Han, and H. Shao-Kang, "A new design of a submicropositioner utilizing electromagnetic actuators and flexure mechanism," IEEE Trans. Ind. Electron., vol. 57, no. 1, pp. 96-106, Jan. 2010.

[13] L. Petit, C. Prelle, E. Dore, F. Lamarque, and M. Bigerelle, "A fourdiscrete-position electromagnetic actuator: Modeling and experimentation," IEEE/ASME Trans. Mechatronics, vol. 15, no. 1, pp. 88-96, Feb. 2010.

[14] S. Hungsun and L. Kok-Meng, "Distributed multipole models for design and control of PM actuators and sensors," IEEE/ASME Trans. Mechatronics, vol. 13, no. 2, pp. 228-238, Apr. 2008.

[15] Y. Liang, I. M. Chen, L. Chee Kian, Y. Guilin, L. Wei, and L. KokMeng, "Design and analysis of a permanent magnet spherical actuator," IEEE/ASME Trans. Mechatronics, vol. 13, no. 2, pp. 239-248, Apr. 2008.

[16] J. Lei, X. Luo, X. Chen, and T. Yan, "Modeling and analysis of a 3-DOF lorentz-force-driven planar motion stage for nanopositioning," Mechatronics, vol. 20, no. 5, pp. 553-565, 2010.

[17] M.-Y. Chen, H.-W. Tzeng, and S.-K. Hung, "A new mechanism design of electro-magnetic actuator for a micro-positioner," ISA Trans., vol. 46, no. 1, pp. 41-48, 2007.

[18] A. T. Al-Halhouli, M. I. Kilani, and S. Büttgenbach, "Development of a novel electromagnetic pump for biomedical applications," Sens. Actuators A, Phys., vol. 162, no. 2, pp. 172-176, 2010.

[19] N.-C. Tsai, J.-S. Liou, C.-C. Lin, and T. Li, "Design of microelectromagnetic drive on reciprocally rotating disc used for microgyroscopes," Sens. Actuators A, Phys., vol. 157, no. 1, pp. 68-76, 2010.
[20] N. Bencheikh, C. Prelle, and F. Lamarque, "Integrated mechatronics device for linear and angular displacements," J. Eur. Syst. Autom. Mecatron., vol. 40, no. 3, pp. 369-388, 2006.

[21] S. A. Janhavi and P. A. David, "A study of scaling and geometry effects on the forces between cuboidal and cylindrical magnets using analytical force solutions," J. Phys. D, Appl. Phys., vol. 41, no. 10, p. 105001, 2008.

[22] M. U. Khan, C. Prelle, N. Bencheikh, F. Lamarque, and S. Büttgenbach, "3 DOF based electromagnetic stage," in Proc. 12th Int. Conf. New Actuators, Bremen, Germany, Jun. 14-16, 2010, pp. 492-496.

[23] M. U. Khan, C. Prelle, F. Lamarque, T. Beutel, and S. Büttgenbach, "Silicon conveyour based electromagnetic device for linear displacement," presented at the IEEE/ASME Int. Conf. Advanced Intelligent Mechatronics Association Information and Management vol. 13, no. 2, Montreal, QC, Canada, Jul. 6-9, 2010.

[24] E. P. Furlani, Permanent Magnet and Electromechanical DevicesMaterials, Analysis and Applications. San Diego, CA: Academic, 2001

[25] C. Prelle, F. Lamarque, and P. Revel, "Reflective optical sensor for longrange and high-resolution displacements," Sens. Actuators A, Phys., vol. 127, no. 1, pp. 139-146, 2006.

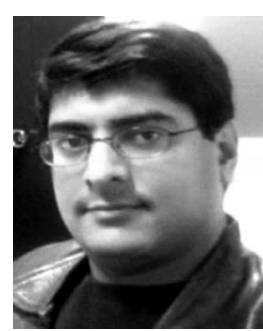

Muneeb Ullah Khan was born in Pakistan in 1981. He received the B.E. degree in mechanical engineering from the University of Engineering and Technology, Peshawar, Pakistan. In 2007, he received his Master's degree in "Design, Industrialization and Innovation" from Art et Métier Paris Tech, Metz, France. He is currently working toward the Ph.D. degree in a joint project between the Roberval Laboratory, Université de Technologie de Compiègne, Compiègne, France, and the Institute for Microtechnology, Technische Universität Braunschweig,

Braunschweig, Germany.

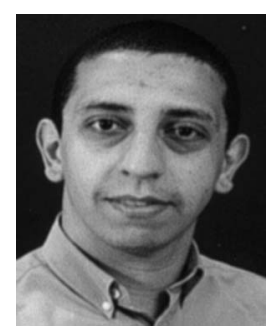

Nabil Bencheikh was born in Algeria in 1974. He received the DEA degree in 2002 and the Ph.D. degree in 2006 in advance mechanics and applications both from the Université de Technologie de Compiègne, Compiègne, France.

Since 2007, he has been with CEDRAT Technology, Meylan, France.

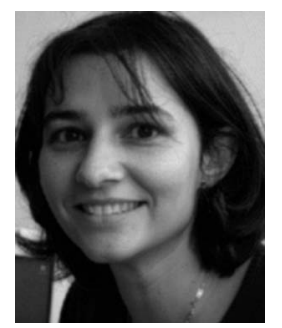

Christine Prelle was born in France in 1971. She received the DEA degree in automatic control from the Université Claude Bernard Lyon 1, Lyon, France, in 1994, and the Ph.D. degree in industrial automatic control from the National Institute for Applied Sciences, Lyon, France, in 1997.

She is currently an Associate Professor of Control Engineering in the Roberval Laboratory, Université de Technologie de Compiègne, Compiègne, France. Her research interests include micromechatronics and control.

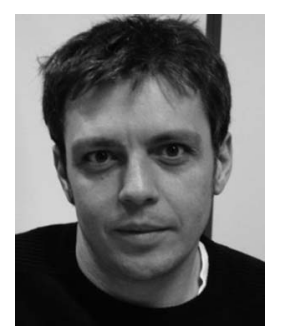

Frédéric Lamarque was born in France in 1971. He received the DEA degree in electronics from ParisSud-Orsay University, Paris, France, from where he also received the Ph.D. degree in electronics in 1998.

$\mathrm{He}$ is currently an Associate Professor at the Université de Technologie de Compiègne, Compiègne, France, in the Roberval Laboratory. His research interests include microsensors and microactuators technologies. 


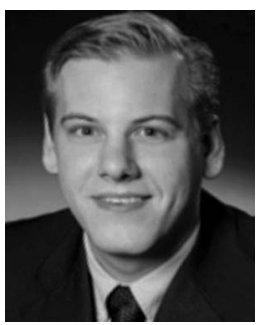

Tobias Beutel received the Diploma degree in the field of aeronautics from the Technische Universität Braunschweig, Braunschweig, Germany, in February 2009 , where he is currently working toward the Doctoral degree in microtechnologies

Since March 2009, he has been working at the Institute for Microtechnology, Technische Universität Braunschweig, as a Researcher focusing on piezoresistive force sensors and pressure sensors for high dynamic measurements in fluids.

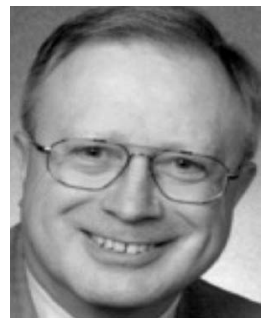

Stephanus Büttgenbach received the Diploma and Ph.D. degrees in physics from the University of Bonn, Bonn, Germany, in 1970 and 1973, respectively.

From 1974 to 1985, he was with the Institute of Applied Physics of the University of Bonn, focusing on atomic and laser spectroscopy. In 1983, he was promoted to Professor of Physics. From 1977 to 1985, he was also a Scientific Associate at CERN, Geneva, Switzerland. In 1985, he joined the HahnSchickard-Society of Applied Research at Stuttgart as the Head of the Department of Microtechnology, where he worked on micromechanics, laser microfabrication, and resonant sensors. From 1988 to 1991, he was the Founding Director of the Institute of Micro and Information Technology of the Hahn-Schickard-Society. In 1991, he became a Professor of Microtechnology at the Technische Universität Braunschweig, Braunschweig, Germany. His current research interests include the development and application of microsensors, microactuators, and microsystems. 